\title{
Oleoylethanolamide inhibits $\alpha$-melanocyte stimulating hormone- stimulated melanogenesis via ERK, Akt and CREB signaling pathways in B16 melanoma cells
}

\author{
Juan Zhou ${ }^{1, *}$, Tong Ren ${ }^{1, *}$, Ying Li ${ }^{2, *}$, Anran Cheng ${ }^{3}$, Wanyi Xie ${ }^{3}$, Lanxi Xu ${ }^{3}$, Lu Peng ${ }^{3}$, \\ Jinbin Lin ${ }^{3}$, Lianxiang Lian ${ }^{3}$, Yong Diao ${ }^{4}$, Xin Jin $^{3}$ and Lichao Yang ${ }^{3}$ \\ ${ }^{1}$ Department of Obstetrics and Gynecology, The First Affiliated Hospital of Xiamen University, Xiamen University, Xiamen, \\ China \\ ${ }^{2}$ Department of Pharmacy, Xiamen Medical College, Xiamen, China \\ ${ }^{3}$ Xiamen Key Laboratory of Chiral Drugs, Medical College, Xiamen University, Xiamen, China \\ ${ }^{4}$ School of Biomedical Sciences, Huaqiao University, Quanzhou, China \\ *These authors have contributed equally to this work and should be considered co-first authors \\ Correspondence to: Xin Jin, email: xinjin@xmu.edu.cn \\ Lichao Yang, email: yanglc116@xmu.edu.cn \\ Keywords: oleoylethanolamide, melanin, tyrosinase, $\alpha$-melanocyte stimulating hormone, B16 mouse melanoma cell \\ Received: January 17, $2017 \quad$ Accepted: April 11, $2017 \quad$ Published: May 23, 2017 \\ Copyright: Zhou et al. This is an open-access article distributed under the terms of the Creative Commons Attribution License 3.0 \\ (CC BY 3.0), which permits unrestricted use, distribution, and reproduction in any medium, provided the original author and source \\ are credited.
}

\section{ABSTRACT}

The present study aimed to examine the potential inhibitory activity of oleoylethanolamide (OEA) on a-melanocyte stimulating hormone (a-MSH)-stimulated melanogenesis and the molecular mechanism(s) involved in the process in B16 mouse melanoma cells. Our data demonstrated that OEA markedly inhibited melanin synthesis and tyrosinase activity in a-MSH-stimulated B16 cells. In addition, the expression of melanogenesis-related proteins, such as melanocortin-1 receptor (MC1R), microphthalmia-associated transcription factor (MITF), tyrosinase-related protein-1 (TRP-1) and tyrosinase, was suppressed in a concentration-dependent manner by OEA. In addition, OEA may suppress melanogenesis through a peroxisome proliferator-activated receptor a (PPARa)-independent pathway. Moreover, OEA activated ERK, Akt, p38 pathways and inhibits CREB pathway in a-MSH-stimulated B16 cells. The specific ERK inhibitor PD98059 partly blocked OEA-inhibited melanin synthesis and tyrosinase activity and partly abrogated the OEA-suppressed expression of melanogenic proteins. Furthermore, OEA presented remarkable inhibition on the body pigmentation in the zebrafish model system. Our findings demonstrated that OEA is an effective inhibitor of hyperpigmentation through activation of ERK, Akt and p38 pathways, inhibition of the CREB pathway, and subsequent down-regulation of MITF, TRP-1 and tyrosinase production.

\section{INTRODUCTION}

Melanogenesis is a physiological process leading to the production of melanin pigment, which plays a vital role in the prevention of sun-induced skin injury and contributes to skin and hair color [1]. However, excessive generation of melanin results in hyperpigmentation, wrinkling, melasma, and other dermatological disorders [2]. Several known melanin synthesis inhibitors, including kojic acid, arbutin and many other natural products, are currently being utilized as cosmetic additives and have already been the focus of studies. However, the applicability of these chemicals might be limited given their adverse side effects. For example, kojic acid can lead to allergic dermatitis, and arbutin might carry carcinogenic risks. Therefore, finding new effective skin whitening products with low adverse effects would be valuable for cosmetic purposes and for clinical treatment of pigmentary disorders caused by melanin accumulation. 
Melanogenesis is a complex process that is regulated by tyrosinase and tyrosinase related protein-1 and -2 (TRP-1 and TRP-2) [3]. Tyrosinase plays a pivotal role in altering melanin generation by the hydroxylation of tyrosine into dihydroxyphenylalanine (DOPA) followed by further oxidation of DOPA into DOPA quinone. Therefore, inhibition of tyrosinase is the most common approach to achieve skin hypopigmentation as it is the key enzyme that catalyzes the rate-limiting step of melanogenesis [4]. In addition, tyrosinase, TRP-1 and TRP-2 are transcriptionally regulated by a crucial transcription factor in melanocytes, namely, microphthalmia-associated transcription factor (MITF) [5]. Skin pigmentation is regulated by a variety of extrinsic and intrinsic factors [6]. In particular, extracellular signal-regulated kinase (ERK) negatively regulates melanogenesis in melanocytes and melanoma cells [7, 8 ] and is also an effective modulator of the activation of MITF, thereby leading to the regulation of melanogenesis.

Peroxisome proliferator-activated receptors (PPARs) are a group of nuclear receptor proteins that are associated with various skin diseases, particularly those involving inflammation, epidermal hyperproliferation, and differentiation $[9,10]$. PPAR $\alpha$ agonists exhibit the potential therapeutic application for the treatment of pigmentary disorders, including vitiligo, melasma and postinflammatory hyperpigmentation [11]. In clinical trials, fewer patients treated with gemfibrozil, a PPAR $\alpha$ agonist, were diagnosed with melanoma compared with the control group [12]. Fenofibrate, another PPAR $\alpha$ agonist, suppresses melanogenesis through activation of the $\mathrm{p} 38$ mitogen-activated protein kinase pathway [13]. Previous studies have demonstrated that oleoylethanolamide (OEA), the potent endogenous ligand of PPAR $\alpha$, possessed antihyperlipidemia and weight reduction properties [14-16]. Moreover, our previous study showed that OEA exhibited potent neuroprotective and anti-inflammatory effects [1719]. Although the positive effects of other PPAR $\alpha$ agonists on inhibiting melanogenesis have been verified, little is known about the anti-melanogenic activity of OEA.

In the present study, we investigated the effects of OEA on the regulation of melanogenesis in $\alpha$-MSHtreated B16 melanoma cells and zebrafish. In addition, we tested whether the PPAR $\alpha$ is a potentially interesting target for OEA in inhibiting melanogenesis. Moreover, the involvement of the molecular mechanism(s) in OEA effects on melanogenesis was also investigated through the inhibition experiments.

\section{RESULTS}

\section{OEA inhibits melanin synthesis and tyrosinase activity in $\alpha$-MSH-stimulated B16 cells at non- cytotoxic dosages}

To avoid the possibility that inhibition of melanogenesis is due to cytotoxicity, we first performed
MTT assays to determine whether OEA is cytotoxic to B16 cells. B16 cells were treated with different concentrations of OEA $(10-100 \mu \mathrm{M})$ for $72 \mathrm{~h}$. The results showed that treatment of OEA caused mild cytotoxicity at the dosage of $80 \mu \mathrm{M}$ (Figure 1B). Thus, 10 to $50 \mu \mathrm{M}$ doses were chosen to determine the effects of OEA on melanogenesis and tyrosinase activity. The results showed that OEA reduced melanin content and tyrosinase activity in a dosedependent manner in $\alpha$-MSH-stimulated B16 cells. At 10 , 30 and $50 \mu \mathrm{M}$ OEA, the melanin content was decreased by $11.99 \%, 13.69 \%$ and $32.00 \%$, respectively (Figure $1 \mathrm{C}-\mathrm{b})$, and tyrosinase activity was decreased by $15.23 \%$, $28.52 \%$ and $32.98 \%$, respectively (Figure $1 \mathrm{C}-\mathrm{c}$ ). Kojic acid, a well-known melanogenesis inhibitor, was used as a positive control. Moreover, the inhibition effect of kojic acid $200 \mu \mathrm{M}$ was similar to $50 \mu \mathrm{M}$ OEA. Meanwhile, we did not observe that OEA inhibits melanin synthesis and tyrosinase activity in normal B16 cells (Supplementary Figure 2).

\section{OEA decreases MC1R, MITF, TRP-1 and tyrosinase protein expression in $\alpha-\mathrm{MSH}-$ stimulated B16 cells}

To determine whether the suppressive activity of OEA is associated with expression levels of melanogenesis-related proteins, such as MCR1, MITF, tyrosinase-related protein-1 (TRP-1), and tyrosinase, cells were exposed to $\alpha$-MSH with or without OEA treatment, and protein extracts were then subjected to Western blot analysis. As shown in Figure 2, MCR1, MITF, TRP-1 and tyrosinase protein expression levels in B16 cells treated with various concentrations of OEA were reduced in a dose-dependent manner compared with control. The data suggest that the inhibition of melanogenesis by OEA is associated with the downregulation of MCR1/MITF-tyrosinase signaling pathways.

\section{PPAR $\alpha$ signaling does not mediate the effect of OEA on melanogenic activity}

To elucidate the mechanism of OEA on melanin production, we sought to determine whether the effects of OEA are mediated through activation of the PPAR $\alpha$ receptor. We also examined the effect of MK886, an inhibitor of PPAR $\alpha$, on melanin levels, tyrosinase activity and tyrosinase expression. We first tested the noncytotoxic concentration in B16 melanoma cells using the MTT assay, and $2 \mu \mathrm{M}$ MK886 was determined as the proper concentration for further study (Figure 3A). The suppression of melanin content, tyrosinase activity and tyrosinase expression by OEA was not altered by MK886 pretreatment (Figure 3B-b, 3C and 3D). In contrast, MK886 could override the OEA-induced repression of $\operatorname{PPAR} \alpha$ (Figure 3E). Thus, these results imply that the biological effects of OEA are not dependent on PPAR $\alpha$ signaling. 


\section{OEA inhibits melanogenesis in $\alpha$-MSH- stimulated B16 cells through activating the ERK pathway}

ERK activation induces the degradation of MITF, which subsequently decreases the expression of tyrosinase [20]. To further understand the inhibition of OEA on melanogenesis, we examined ERK and p-ERK protein levels in $\alpha$-MSH stimulated B16 cells. Our results demonstrated that $\alpha$-MSH stimulation reduced the levels of p-ERK1/2 in B16 cells. Therefore, this result indicates that ERK1/2 is involved in melanogenesis in B16 cells. However, OEA significantly increased p-ERK1/2 levels in a dose-dependent manner (Figure 4A-a). Total ERK1/2 levels exhibited no difference among the groups (Figure

A

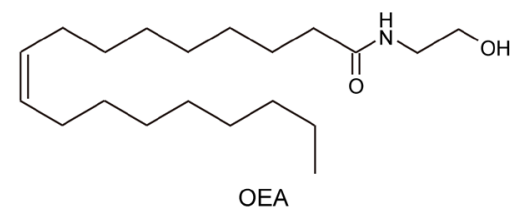

C

a

b
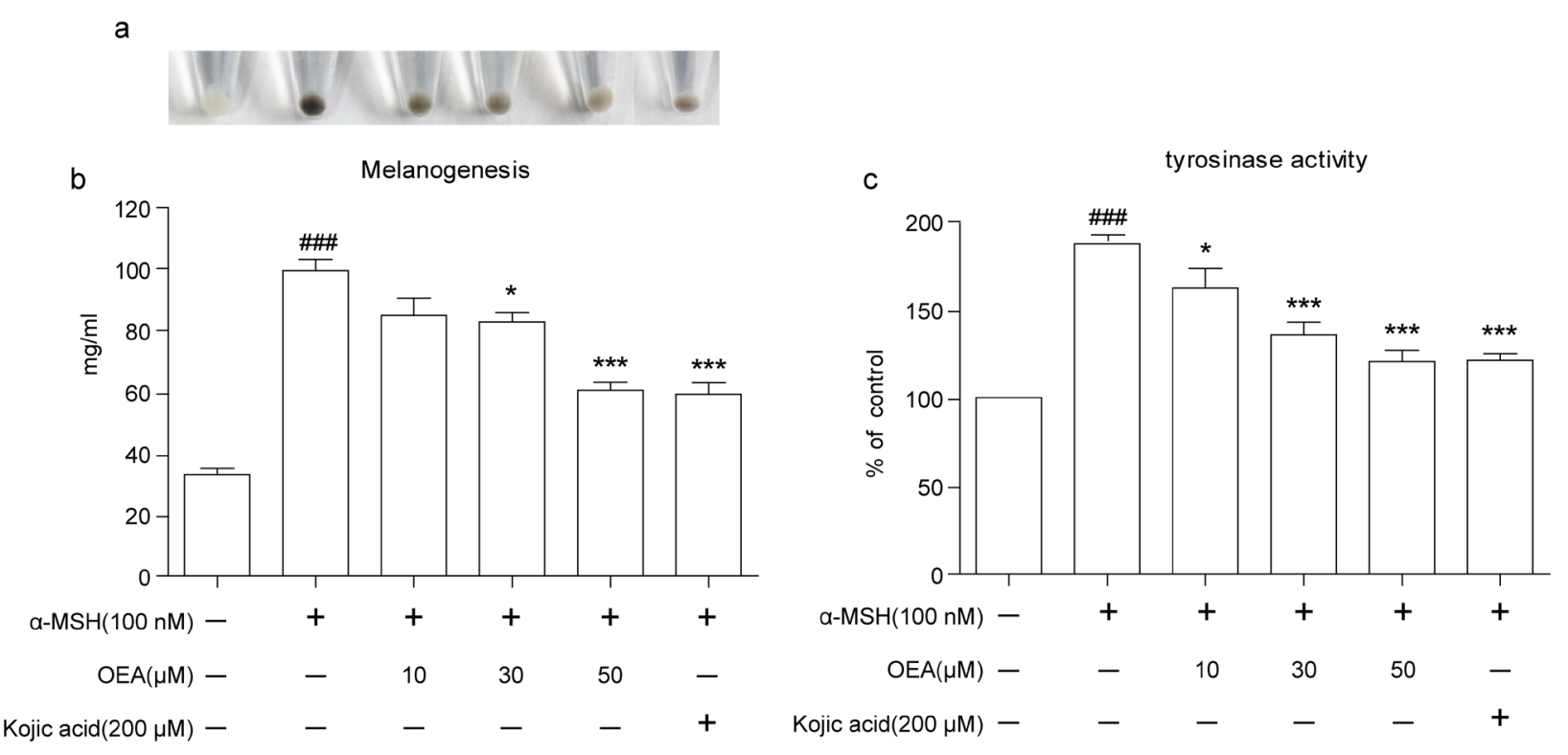

4A-b). Thus, our results indicated that ERK1/2 signaling is associated with the effects of OEA on melanogenesis alteration in B16 cells.

To further confirm the role of the ERK pathway on the OEA-induced anti-melanogenic effect, we used a specific inhibitor of ERK, PD98059, which blocks ERK signaling. Cells were pre-treated with PD98059 in the presence of $\alpha-\mathrm{MSH}$ and OEA, and then we measured melanin levels and tyrosinase activity. The results indicated that the synergistic effect of $\alpha$-MSH and OEA on melanin production and tyrosinase activity was partially offset by PD98059 treatment (Figure 4B-a and 4B-b). Additionally, to confirm our results, we further examined the effect of PD98059 on the expression of MITF, TRP1 and tyrosinase in the presence of $\alpha$-MSH or OEA.

B

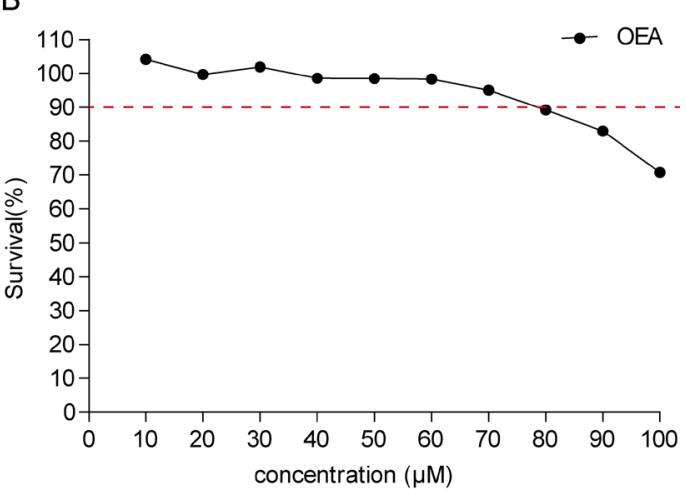

Figure 1: Effect of OEA on cellular melanin synthesis and tyrosinase activity in $\alpha$-MSH-stimulated B16 cells. (A) The chemical structure of OEA. (B) Cells were treated with various concentrations of OEA for $72 \mathrm{~h}$. Cell viability was determined by MTT assay. (C) Relative cellular melanin content and tyrosinase activity were measured at $72 \mathrm{~h}$ after treatment. Cells were exposed to $100 \mathrm{nM}$ $\alpha$-MSH in the presence of 10 to $50 \mu \mathrm{M}$ OEA or $200 \mu \mathrm{M}$ kojic acid. The percentage values of the treated cells are expressed relative to that in control cells. Kojic acid was used as a positive control. Data are reported as the mean $\pm \mathrm{SEM}$ of three independent experiments performed in triplicate $(\mathrm{n}=3) .{ }^{\# \#} P<0.001$ vs. control group; ${ }^{*} P<0.05,{ }^{* * *} P<0.001$ vs. $\alpha$-MSH-stimulated group. 
PD98059 partially restored OEA-induced MITF, TRP-1, and tyrosinase down-regulation in $\alpha$-MSH-stimulated B16 cells (Figure 4B-c, 4B-d and 4B-e). We next examined whether PD98059 inhibits the ERK pathway in $\alpha-\mathrm{MSH}-$ stimulated B16 cells and found that PD98059 does inhibit ERK activation in OEA-treated B16 cells (Figure 4C). Therefore, our results imply that OEA-induced inhibition of melanogenesis through activating the ERK signaling pathways.

\section{Akt, CREB and p38 signaling pathways are involved in the suppressive effect of OEA on melanogenesis}

To further elucidate the mechanisms underlying the anti-melanogenic effect of OEA, we examined whether OEA influences Akt, CREB and/or p38 activation. As shown in Figure 5 and Supplementary Figure 1, OEA induces Akt and p38 phosphorylation but inhibits CREB

B

MITF

$\beta$-actin
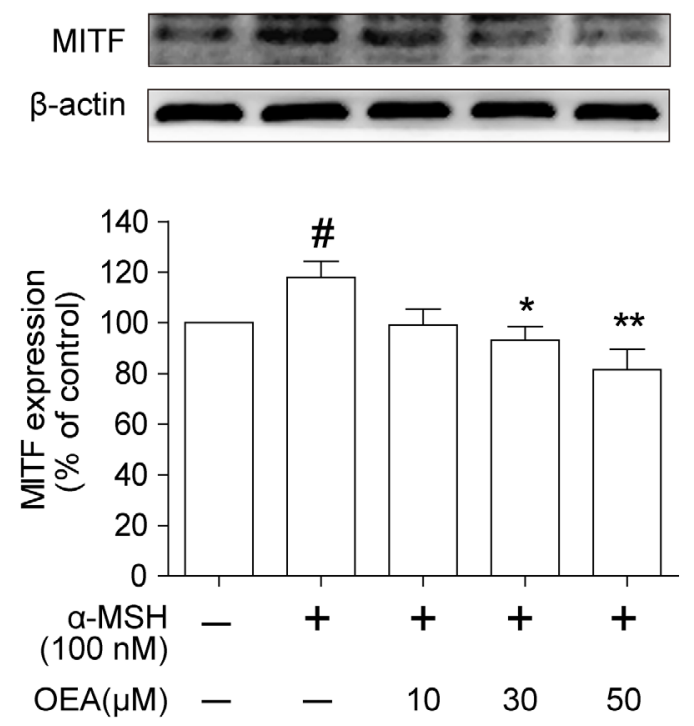

D
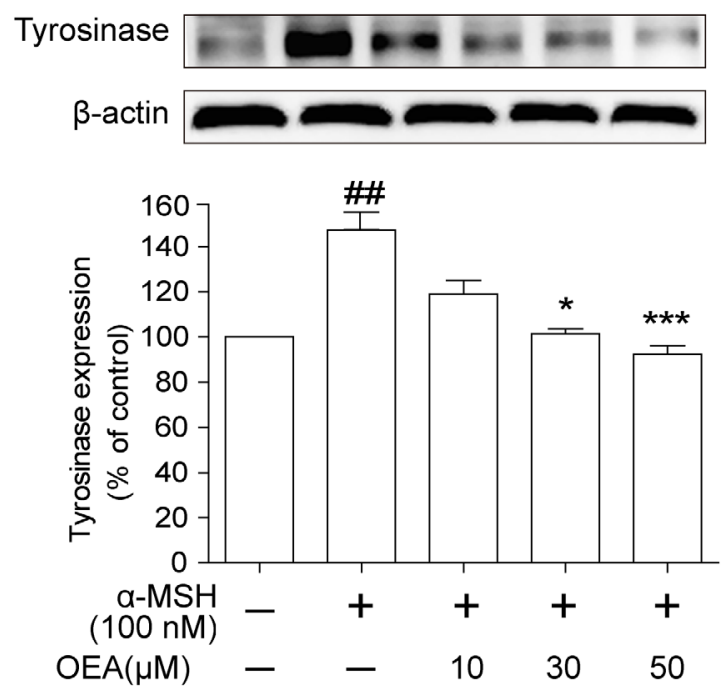

Figure 2: Effect of OEA on the expression of melanogenesis-related proteins in $\alpha$-MSH-stimulated B16 cells. Cells were exposed to $100 \mathrm{nM} \alpha-\mathrm{MSH}$ in the presence of 10 to $50 \mu \mathrm{M}$ OEA for $72 \mathrm{~h}$. (A) MC1R, (B) MITF, (C) TRP-1 and (D) tyrosinase protein levels were examined by Western blot. The data are presented as percentages compared with the control group (set to $100 \%$ ) and represented as the means \pm SEM of three separate experiments performed in duplicate $(\mathrm{n}=3)$. ${ }^{\#} P<0.05,{ }^{\#} P<0.01,{ }^{\# \#} P<0.001$ vs. control group; ${ }^{*} P<0.05$, ${ }^{* *} P<0.01,{ }^{* * *} P<0.001$ vs. $\alpha$-MSH-stimulated group. 
phosphorylation in a dose-dependent manner. Total Akt, CREB and p38 levels did not differ among the groups. Thus, these results imply that OEA-induced inhibition of melanogenesis occurs through Akt, CREB and p38 signaling pathways.

\section{OEA suppresses melanogenesis in zebrafish}

Zebrafish has melanin pigments on the surface, allowing simple observation of the pigmentation process without complicated experimental procedures. Therefore, we further tested the effects of OEA on zebrafish pigmentation. We used PTU as a positive control, which is a sulfur-containing tyrosinase inhibitor that is used widely in zebrafish research [21]. As shown in Figure 5A,
$150 \mu \mathrm{M}$ OEA produced remarkable inhibition on body pigmentation. Compared with untreated embryos, 100 and $150 \mu \mathrm{M}$ OEA exposure resulted in $33.5 \pm 1.66 \%$ and $50.5 \pm 4.16 \%$ reduction in melanogenesis, respectively. In addition, $100 \mu \mathrm{M}$ PTU and $100 \mu \mathrm{M}$ and $150 \mu \mathrm{M}$ OEA showed no significant effect on embryo survival rate (Figure 6B). When assessing morphological malformations, OEA did not exhibit conspicuous adverse effects (data not shown).

\section{DISCUSSION}

A previous report demonstrated that OEA is a potent endogenous ligand of PPAR $\alpha$ [14]. The administration of OEA, as a pharmacological drug, modulates feeding, lipid

B a
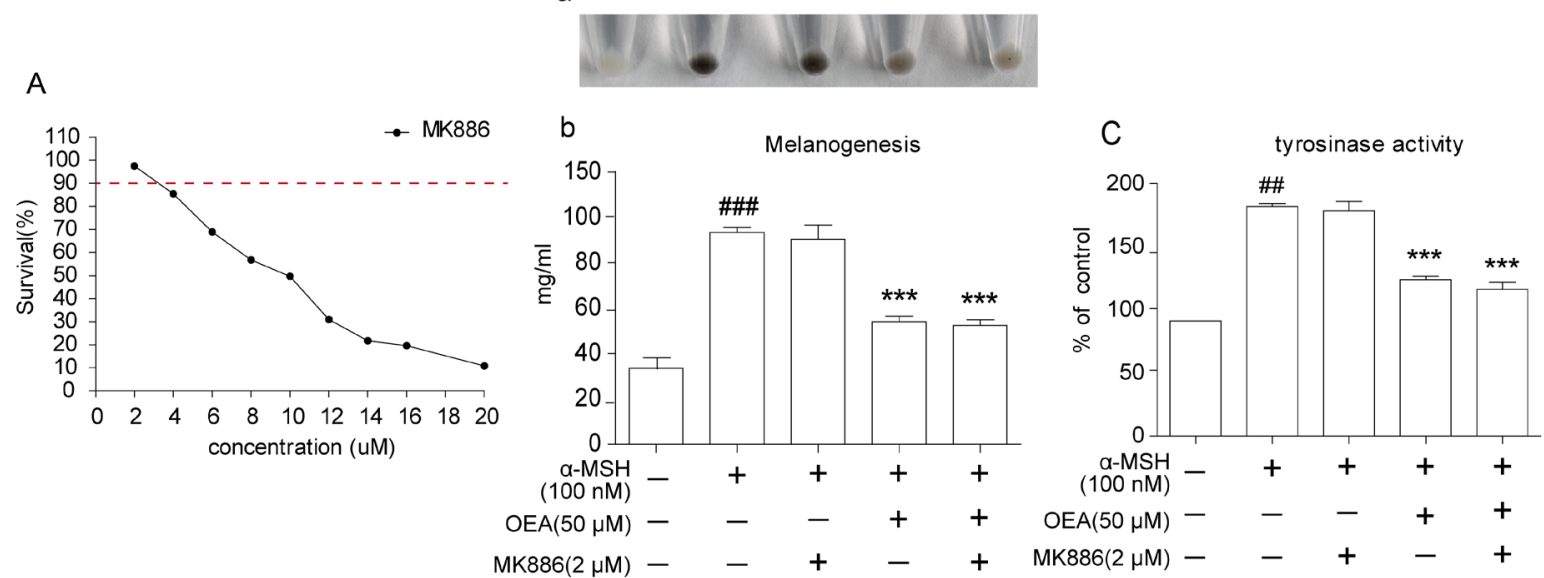

D
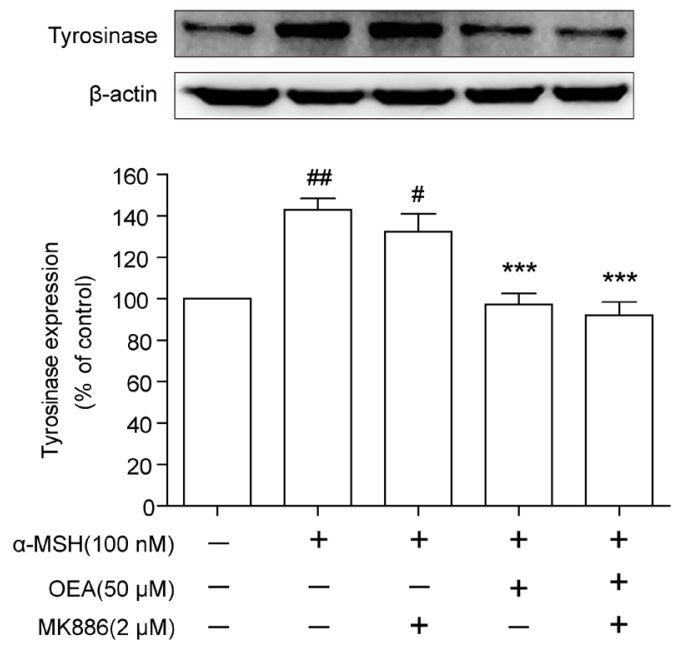

$\mathrm{E}$
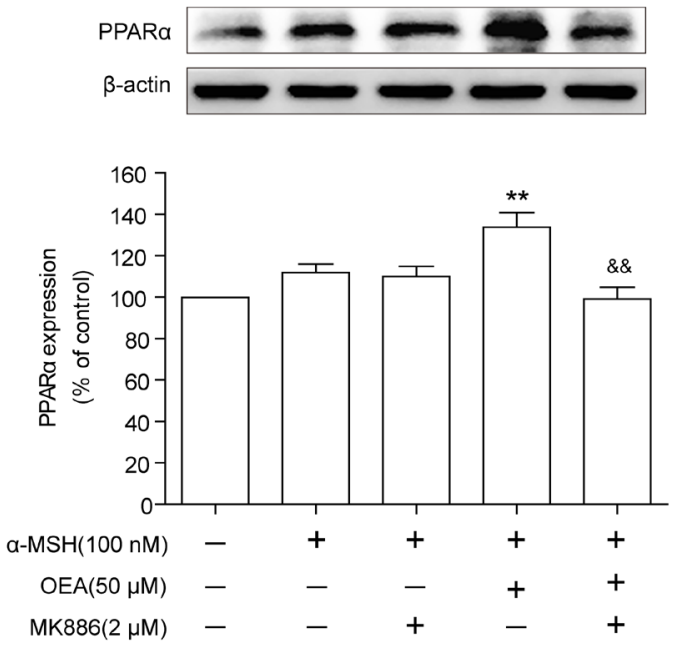

Figure 3: Effects of OEA on the PPAR $\alpha$ signaling in $\alpha$-MSH-stimulated B16 cells. (A) Cells were treated with various concentrations of MK886 for $72 \mathrm{~h}$. B16 cells were pretreated with DMSO only or MK886 $(2 \mu \mathrm{mol} / \mathrm{L})$ for $1 \mathrm{~h}$ followed by the addition of 100 $\mathrm{nM} \alpha-\mathrm{MSH}$ in the absence or in the presence of $50 \mu \mathrm{M}$ OEA. (B) Relative melanin content and (C) tyrosinase activity were measured, and (D and E) the cell lysates were isolated through Western blot at $72 \mathrm{~h}$ after OEA treatment. The data are presented as percentages compared with the control group (set to $100 \%$ ) and represented as the means \pm SEM of three separate experiments performed in duplicate ( $\mathrm{n}=3$ ). ${ }^{\#} P<0.05,{ }^{\#} P<0.01,{ }^{\# \#} P<0.001$ vs. control group; ${ }^{* *} P<0.01,{ }^{* * *} P<0.001$ vs. $\alpha-M S H-$ stimulated group; ${ }^{\&} P<0.01$ vs. $\alpha-M S H+O E A$ group. 


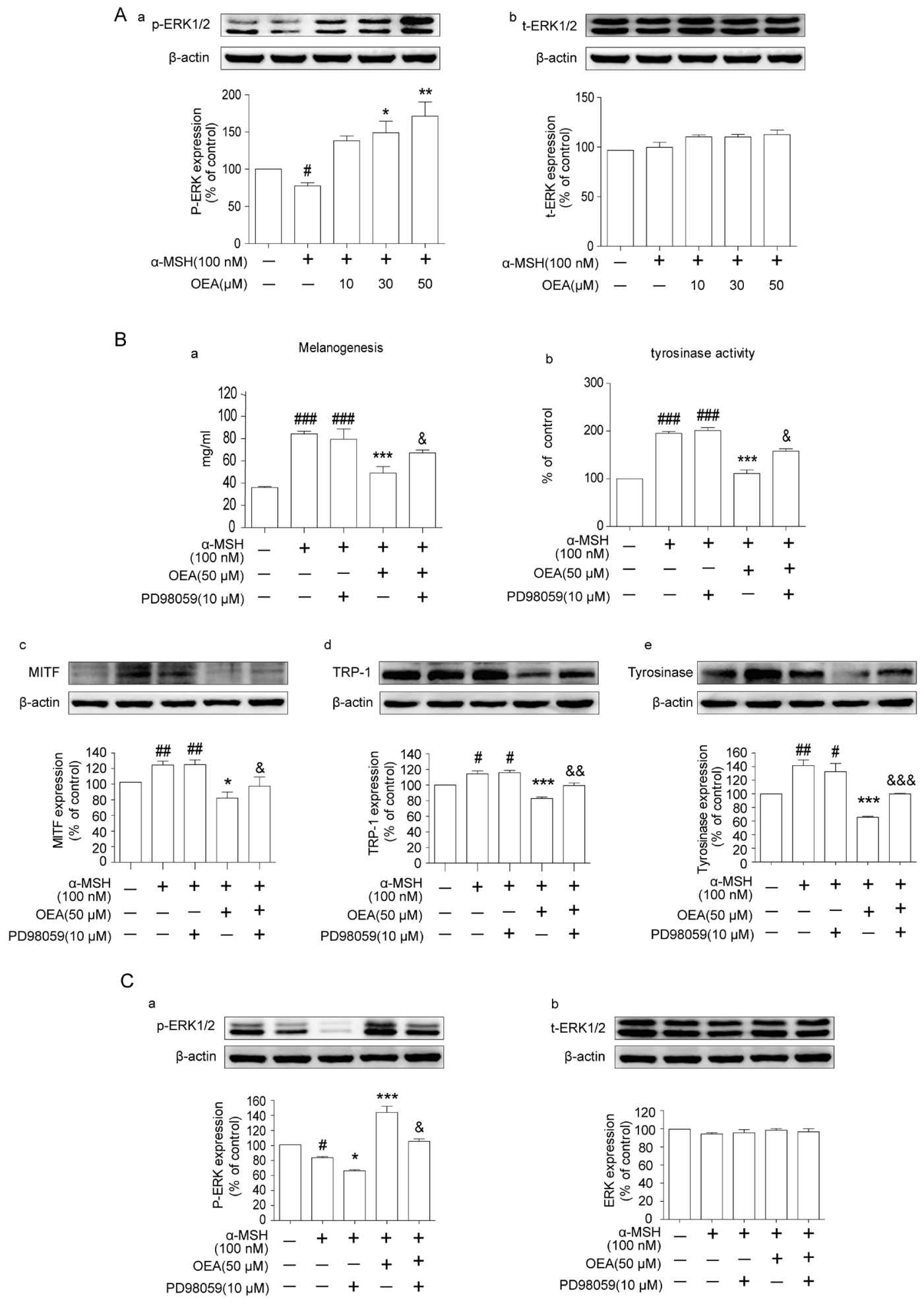

Figure 4: Effects of OEA on the ERK signaling pathway in $\boldsymbol{\alpha}$-MSH-stimulated B16 cells. B16 cells were pretreated with DMSO only or PD98059 (10 $\mu \mathrm{mol} / \mathrm{L})$ for $1 \mathrm{~h}$ followed by the addition of $100 \mathrm{nM} \alpha-\mathrm{MSH}$ in the absence or in the presence of $50 \mu \mathrm{M}$ OEA. (B-a) Relative melanin content and (B-b) tyrosinase activity were measured, and (A, B-c, B-d, B-e, and C) the cell lysates were isolated through Western blot at $72 \mathrm{~h}$ after OEA treatment. The data are presented as percentages compared with the control group (set to $100 \%$ ) and represented as the means \pm SEM of three separate experiments performed in duplicate $(\mathrm{n}=3) .{ }^{\#} P<0.05,{ }^{\# \#} P<0.01,{ }^{\# \#} P<0.001 \mathrm{vs}$. control group; ${ }^{*} P<0.05,{ }^{* *} P<0.01,{ }^{* * *} P<0.001$ vs. $\alpha$-MSH-stimulated group; ${ }^{\&} P<0.05$, ${ }^{\&} P<0.01$, \&\&\& $P<0.001$ vs. $\alpha$-MSH+OEA group. 
metabolism, glucose homeostasis, and anti-atherosclerotic functions through activation of the PPAR $\alpha$ signaling pathway $[14,16,22]$. Furthermore, our previous study showed that OEA exhibited potent neuroprotective and anti-inflammatory effects [17-19]. However, despite numerous published results, the effect of OEA on melanogenesis has not been reported. In the present study, we investigated the effect of OEA on melanin synthesis and the molecular mechanism(s) involved in the process in B16 mouse melanoma cells.

Melanin levels directly correlate with the protein levels of tyrosinase and the activity of tyrosinase [23]. We first investigated whether OEA could inhibit melanin synthesis in the presence of $\alpha-\mathrm{MSH}$. Kojic acid is a kind of melanoma specific inhibitor, it changes the threedimensional structure of tyrosinase and prevents tyrosinase activation, and then inhibits the formation of melanin [24]. Therefore, kojic acid was used as a positive control in the vitro study. We found that OEA inhibited melanin synthesis and tyrosinase activity in a concentrationdependent manner without cytotoxicity $(10-50 \mu \mathrm{M})$ in B16 cells and that the extent of inhibition was comparable between treatment with OEA $(50 \mu \mathrm{M})$ and with kojic acid $(200 \mu \mathrm{M})$. These results demonstrate that OEA inhibits melanin synthesis and down-regulates tyrosinase activity in $\alpha$-MSH-stimulated B16 cells. In general, through binding to MC1R, $\alpha$-MSH potently induces MITF expression, which controls pigmentation by regulating the

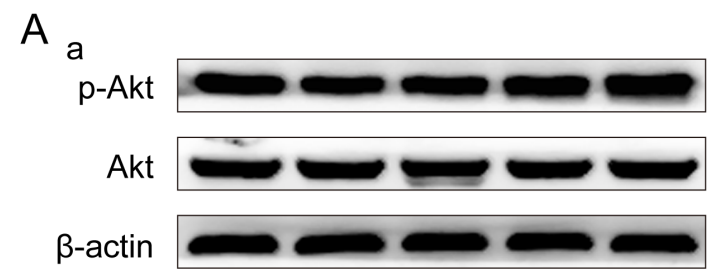

b
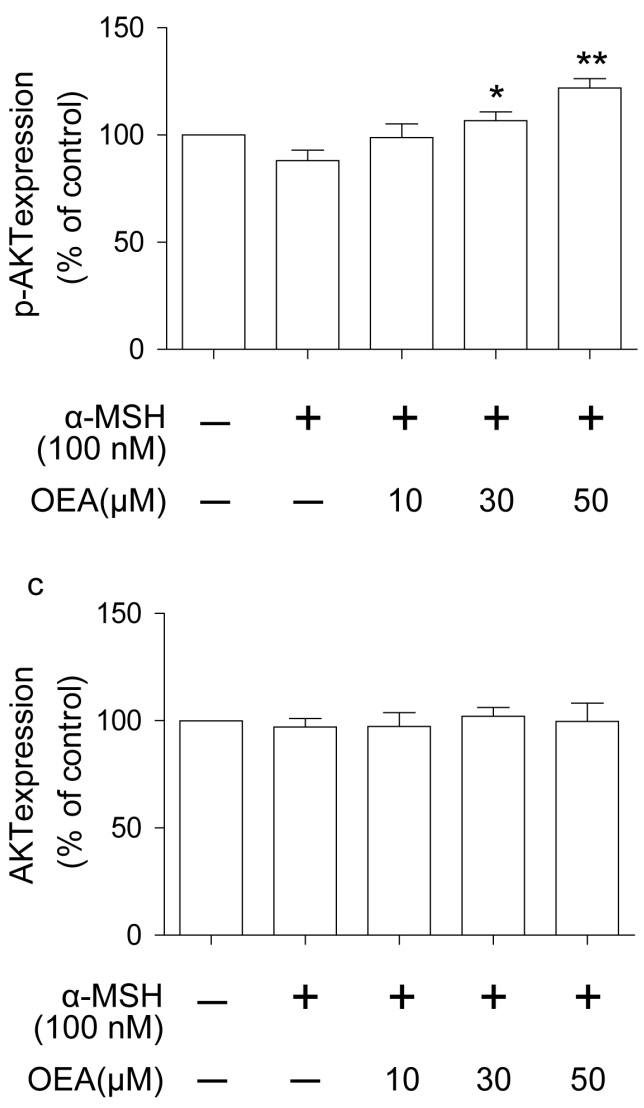

$\mathrm{B}_{\mathrm{a}}$

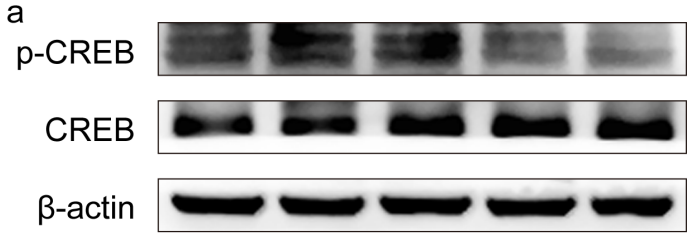

b
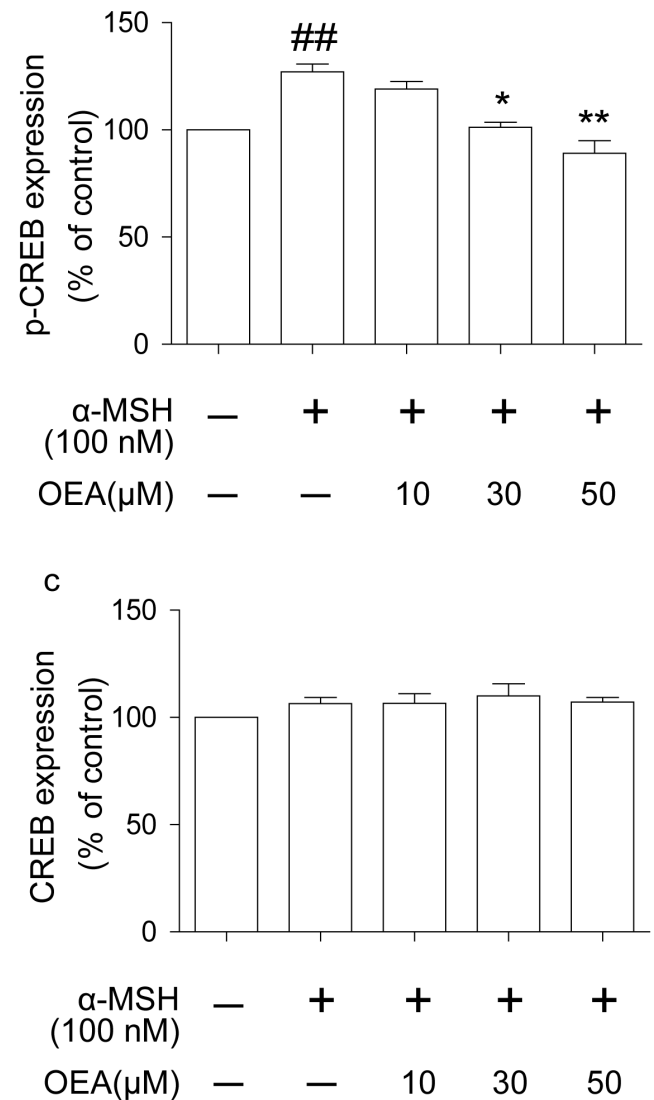

Figure 5: Effect of OEA on Akt and CREB signaling pathways in $\alpha$-MSH-stimulated cells. (A-a and B-a) The protein levels of p-Akt, Akt, p-CREB, and CREB were examined by Western blot. (A-b, A-c, B-b, and B-c) The data are presented as percentages compared with the control group (set to $100 \%$ ) and represented as the means \pm SEM of three separate experiments performed in duplicate (n=3). ${ }^{\#} P<0.01$ vs. control group; ${ }^{*} P<0.05,{ }^{* *} P<0.01$ vs. $\alpha$-MSH-stimulated group. 
expression of melanogenic enzymes, such as TRP-1, TRP2 , and tyrosinase, that increase melanin synthesis [25]. Therefore, it is important to verify the inhibitory effect of OEA on melanogenesis by assessing the down-regulation of MITF and TRP expression. Our results indicate that OEA significantly reduces MC1R, MITF, TRP-1 and tyrosinase protein levels in $\alpha$-MSH-stimulated B16 cells. These data suggest that OEA does not directly inhibit tyrosinase activity and that OEA's inhibitory effect results in MITF and TRPs down-regulation in B16 cells.

PPAR $\alpha$ is a ligand-activated transcription factor that is involved in various skin diseases, such as psoriasis, acne, atopic dermatitis, scleroderma and melanoma [26]. PPAR $\alpha$ is a major research target for the understanding and treatment of numerous skin pathologies, including hyperproliferative and inflammatory diseases. To evaluate whether the suppressive melanogenesis of OEA was induced through PPAR $\alpha$, we examined the effect of MK886, an inhibitor of PPAR $\alpha$ on melanin levels, tyrosinase activity and tyrosinase expression. Our results revealed that OEA reduces melanin content and tyrosinase activity, and these phenomena were not reversed by the PPAR $\alpha$ antagonist MK886. In addition, OEA increased PPAR $\alpha$ protein expression in $\alpha$-MSH-stimulated B1 6 cells, but this effect was almost abrogated by MK886 treatment. Our results imply that the biological effects of OEA are

A
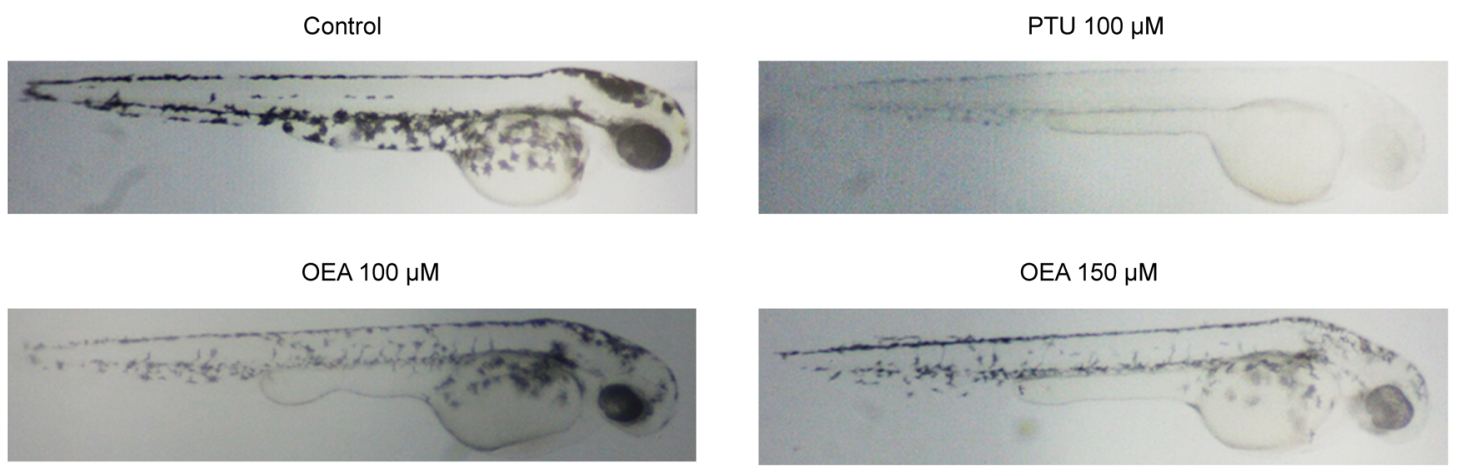

B

\begin{tabular}{ccc}
\hline Drug & Embryon (Die/Sum) & Inhibition Ratio(\%) \\
\hline PTU $(100 \mu \mathrm{M})$ & $0 / 10$ & $97.4 \pm 3.52$ \\
OEA $(100 \mu \mathrm{M})$ & $0 / 10$ & $33.5 \pm 1.66$ \\
OEA $(150 \mu \mathrm{M})$ & $0 / 10$ & $50.5 \pm 4.16$ \\
\hline
\end{tabular}

Figure 6: Effects of OEA on melanogenesis in zebrafish. (A) Representative photographs of zebrafish. Synchronized embryos were treated with melanogenic inhibitors at the indicated concentrations. The compounds were dissolved in $0.05 \%$ DMSO and then added to the embryo medium. The effects on the pigmentation of zebrafish were observed under the stereomicroscope. (B) Melanin pigment. Approximately 10 embryos were collected, and the stereomicroscope was used to observe the effects on the pigmentation of zebrafish at 48 hours post fertilization. Images capturing and pixel measurement analysis were performed by ImageJ and Photoshop. 
tyrosinase protein down-regulation was also partially reversed by PD98059 in $\alpha-\mathrm{MSH}$-stimulated B16 cells. These results suggests that OEA inhibits melanin synthesis and tyrosinase activity through ERK signaling pathwaymediated suppression of MITF, TRP-1 and tyrosinase in $\alpha$-MSH-stimulated B16 cells. Therefore, our results further confirmed that the ERK signaling pathway plays an essential role in the regulation of melanogenesis.

Our results also indicate that there may be other pathway(s) involved in the inhibition of melanin synthesis by OEA because the anti-melanogenic effect of OEA was not completely abrogated by inhibition of the ERK pathway. Transcriptional regulation of tyrosinase expression is mainly dependent on MITF, which is upregulated by CREB and down-regulated by $\mathrm{PI}$ KK/Akt. Activation of the PI3K/Akt pathway reduces tyrosinase transcription and melanogenesis in B16F10 cells [28]. The p38 mitogen-activated protein kinase (MAPK) pathway is also involved in the regulation of melanogenesis [29]. CREB is an important regulator of MITF. Once phosphorylated, CREB up-regulates MITF, which binds to M-box and E-box motifs in the promoter of target genes including melanogenesis for transcriptional up-regulation of the key enzyme in melanin production [30]. Therefore, we further examined whether OEA activates/inhibites Akt, CREB and p38 pathways. In the present study, our results showed that OEA significantly increased the activity of Akt and p38 but inhibited CREB activation. These results suggest that OEA-induced Akt, CREB and p38 signalings may also play an important role in melanogenesis regulation in B16 cells.

Furthermore, we tested the effects of OEA on the pigmentation of zebrafish. Zebrafish is a highly advantageous vertebrate model organism given its similar gene sequences and organ systems to humans. In the present study, the results showed that OEA decreased body pigmentation. OEA was determined to effectively suppress the production of melanin in vitro and inhibit body pigmentation of zebrafish in vivo. Therefore, one interesting finding of our study is the consensus between the results obtained from in vitro and in vivo experiments.

In summary, our findings demonstrated that OEA inhibits melanin synthesis and tyrosinase activity through ERK pathway-mediated suppression of MITF and TRP1 in $\alpha$-MSH-stimulated B16 cells. In addition, OEAinduced anti-melanogenesis may also be associated with Akt, CREB and p38 signaling pathways regulation (Figure 7). Moreover, OEA exerts an inhibitory effect on

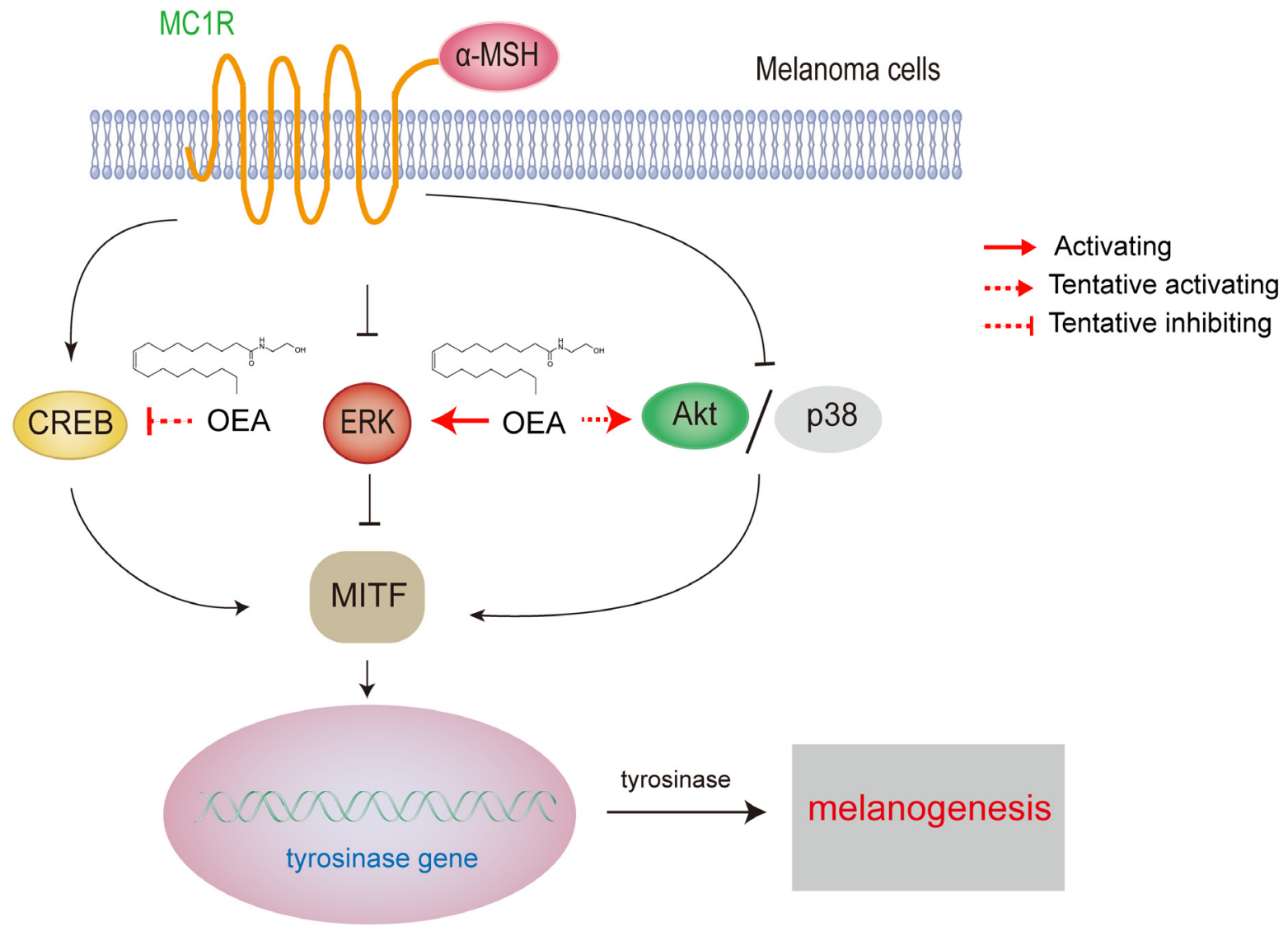

Figure 7: The mechanism of OEA inhibition of $\alpha$-melanocyte stimulating hormone-stimulated melanogenesis in B16 melanoma cells. 
melanogenesis through a PPAR $\alpha$-independent pathway. Therefore, OEA may be a useful therapeutic agent for use in the treatment of hyperpigmentation and may be an effective component in whitening and lightening cosmetics.

\section{MATERIALS AND METHODS}

\section{Materials}

Dulbecco's modified Eagle's medium (DMEM), fetal bovine serum (FBS), penicillin and streptomycin were purchased from Gibco BRL (Carlsbad, CA, USA). OEA, kojic acid, MK886, L-DOPA, dimethyl sulfoxide (DMSO), 3-(4,5-dimethylthiazol-2-yl)-2,5diphenyltetrazolium bromide (MTT), $\alpha$-melanocytestimulating hormone $(\alpha-\mathrm{MSH}), \mathrm{PD} 98059$ and PTU were purchased from Sigma-Aldrich Co. (St Louis, MO, USA). Antibodies against MC1R (1:1000, Cell Signaling Technology, Boston, USA), MITF (1:1000, Cell Signaling Technology), TRP-1 (1:1000, Cell Signaling Technology), Tyrosinase (1:1000, Cell Signaling Technology), PPAR $\alpha$ (1:1000, Abcam, Cambridge, UK), total-ERK (1:1000, Cell Signaling Technology), phospho-ERK (Thr202/Thr204) (1:1000, Cell Signaling Technology), total-Akt (1:1000, Cell Signaling Technology), phospho-Akt (Ser473) (1:1000, Cell Signaling Technology), total-CREB (1:1000, Cell Signaling Technology), phospho-CREB (Ser133) (1:1000, Cell Signaling Technology), total-p38 (1:1000, Cell Signaling Technology), phospho-p38 (1:1000, Cell Signaling Technology) and mouse monoclonal anti- $\beta$ actin $(1: 10000$, Sigma) were obtained from the indicated manufacturers. OEA, kojic acid, MK886, PD98059 and PTU were dissolved in dimethyl sulfoxide (DMSO), and the final DMSO concentration was less than $0.05 \%$. L-DOPA was dissolved in phosphate buffered saline (PBS).

\section{Cell culture}

Murine B16 melanoma cells were obtained from the Type Culture Collection of the Chinese Academy of Sciences, Shanghai, China. Cells were grown in DMEM supplemented with $10 \%$ heat-inactivated FBS and $1 \%$ penicillin/streptomycin at $37^{\circ} \mathrm{C}$ in a humidified atmosphere of $5 \% \mathrm{CO}_{2}$. For experiments, melanocytes were used from passages 2 to 10 .

\section{Cell viability assay}

To examine the effect of OEA on cell viability, the MTT assay was performed according to the manufacturer's instructions. Briefly, B16 cells were seeded in 96-well plates $\left(5 \times 10^{3}\right.$ cells/well $)$ and allowed to adhere at $37^{\circ} \mathrm{C}$ for $24 \mathrm{~h}$. Cells were then treated with various concentrations of OEA for $72 \mathrm{~h}$. After treatment, $100 \mu \mathrm{l}$ of MTT solution ( $5 \mathrm{mg} / \mathrm{ml}$ in PBS) was added to each well, and cells were incubated at $37^{\circ} \mathrm{C}$ for $4 \mathrm{~h}$. Following medium removal, $100 \mu \mathrm{l}$ of DMSO was added to each well, and plates were gently shaken for $5 \mathrm{~min}$. Optical absorbance was determined at $490 \mathrm{~nm}$ with a microplate spectrophotometer (BD Bioscience, USA). Absorbance of cells without treatment was regarded as $100 \%$ of cell survival. Each treatment was performed in triplicate, and each experiment was repeated thrice.

\section{Measurement of cellular melanin contents}

The effect of OEA on $\alpha$-MSH-induced melanogenesis in B16 cells was investigated according to a previously published protocol with slight modifications [31]. Briefly, B16 cells were cultured at $1 \times 10^{5}$ cells $/ \mathrm{ml}$ in 6 -well plates as described above for $24 \mathrm{~h}$. Then, the cells were treated with $\alpha$-MSH $(100 \mathrm{nM})$ in the absence and presence of different concentrations of OEA $(10,30$, and $50 \mu \mathrm{M})$ or kojic acid $(200 \mu \mathrm{M})$ for an additional $72 \mathrm{~h}$. After treatment, cells were washed in ice-cold PBS and dissolved in $1 \mathrm{ml}$ of $1 \mathrm{M} \mathrm{NaOH}$ containing $10 \%$ DMSO at $90^{\circ} \mathrm{C}$ for $60 \mathrm{~min}$. Then, absorbance was measured at $405 \mathrm{~nm}$ using the SpectraMax M5 microplate reader (Molecular Devices Corp., Sunnyvale, CA). To measure the amount of melanin in the experiment, the rate of inhibition in the treatment groups was calculated by the absorbance of known concentrations of synthetic melanin, correcting for the total amounts of protein that are present in the supernatants of cell lysates [32].

\section{Measurement of cellular tyrosinase activity}

Tyrosinase activity was estimated by measuring the rate of production of dopachrome from L-DOPA. B16 cells were treated as described above. Then, the cells were treated with $\alpha$-MSH $(100 \mathrm{nM})$ in the absence and presence of different concentrations of OEA $(10,30$, and $50 \mu \mathrm{M})$ or kojic acid $(200 \mu \mathrm{M})$ for an additional $72 \mathrm{~h}$. After treatment, cells were washed twice with PBS and homogenized with $150 \mu \mathrm{l}$ ice-cold PBS containing 1\% (w/v) Triton X-100 (Sigma) and $0.1 \mathrm{mM}$ phenylmethanesulfonylfluoride (PMSF) by freezing and thawing. The lysates were clarified by centrifugation at $13,000 \mathrm{r} / \mathrm{min}$ for $20 \mathrm{~min}$, and then supernatants were collected. Afterward, the cellular extracts $(90 \mu \mathrm{l})$ were transferred into a freshly prepared $10 \mu \mathrm{l}$ L-DOPA solution ( $0.25 \%$ in PBS) in a well of a $96-$ well plate and incubated in the dark for $1 \mathrm{~h}$ at $37^{\circ} \mathrm{C}$. After incubation, the generated dopachrome was monitored by an ELISA reader at the absorbance of $405 \mathrm{~nm}$ using the SpectraMax M5 microplate reader (Molecular Devices Corp., Sunnyvale, CA). Protein quantification of each lysate was performed using the BCA protein assay kit. For data analysis, the activity was calculated in units divided by the amount of protein measured. 


\section{Western blot analysis}

The protein samples $(30 \mu \mathrm{g})$ were separated on $10 \%$ SDS-PAGE gels and transferred onto polyvinylidene difluoride membranes (Millipore, Billerica, MA, USA). The membranes were blocked with 5\% BSA in Trisbuffered saline containing $0.1 \%$ Tween 20 and incubated with specific antibodies. The values were normalized to the $\beta$-actin intensity levels. The immunoblots were detected with enhanced chemiluminescence reaction reagents, and the images were captured with Image Station 4000R (Rochester, New York, USA).

\section{Effects on zebrafish embryo pigmentation}

In addition to estimating the effects of OEA on melanogenesis in vivo, we further evaluate its antipigmentation ability in zebrafish according to the previous method with slight modification [33]. Embryos were obtained from natural spawning (School of life sciences, Xiamen University), which was induced in the morning by turning on the light. Briefly, 10 to 15 zebrafish embryos were transferred into 24 -well plates, which were maintained in $1 \mathrm{ml}$ embryo medium solution with a 14/10 hour light/dark cycle at $28.5^{\circ} \mathrm{C}$. OEA was dissolved in DMSO and added to the embryo medium at the final concentration of 100 and $150 \mu \mathrm{M}$ from 9 to 48 hours postfertilization (h.p.f.) (39 hours exposure). In addition, 100 $\mu \mathrm{M}$ PTU considered as a standard positive control. To ensure drug stability, the dressing was changed every 12 $\mathrm{h}$ during this period. A stereomicroscope was employed to observe the effects on the pigmentation of zebrafish at 48 h.p.f. Image capturing and pixel measurement analysis were performed using ImageJ and Photoshop. The quantification of pigmentation data was calculated as the percentage change in comparison with the control group, which was considered as $100 \%$.

\section{Statistical analysis}

Results are expressed as the means \pm standard error (SEM). Data were analyzed by one-way analysis of variance (ANOVA) of the differences within treatments followed by Tukey's post hoc test (Prism 5 for Windows, GraphPad Software Inc., USA). $P<0.05$ was considered statistically significant.

\section{Author contributions}

L.Y. and X.J. conceived and designed the experiments. J. Z., T. R., Y. L., A. c., W. X., L. X., L. P and J. L. performed the experiments. Y. L. and L. L. analysed the data. L.Y., Y.D. and J. Z. wrote the paper. All authors reviewed the manuscript.

\section{ACKNOWLEDGMENTS}

This study was supported by grants from the Natural Science Foundation of Fujian, China (No. 2016J01415 and 2016D024).

\section{CONFLICTS OF INTEREST}

The authors declare that they have no conflicts of interest.

\section{REFERENCES}

1. Gilchrest BA, Eller MS. DNA photodamage stimulates melanogenesis and other photoprotective responses. J Investig Dermatol Symp Proc. 1999; 4:35-40.

2. Jadotte YT, Schwartz RA. Melasma: insights and perspectives. Acta Dermatovenerol Croat. 2010; 18:124-129.

3. Fang D, Kute T, Setaluri V. Regulation of tyrosinase-related protein-2 (TYRP2) in human melanocytes: relationship to growth and morphology. Pigment Cell Res. 2001; 14:132-139.

4. Solano F, Briganti S, Picardo M, Ghanem G. Hypopigmenting agents: an updated review on biological, chemical and clinical aspects. Pigment Cell Res. 2006; 19:550-571.

5. Ye Y, Chu JH, Wang H, Xu H, Chou GX, Leung AK, Fong WF, Yu ZL. Involvement of p38 MAPK signaling pathway in the anti-melanogenic effect of San-bai-tang, a Chinese herbal formula, in B16 cells. J Ethnopharmacol. 2010; 132:533-535.

6. Yamaguchi Y, Hearing VJ. Physiological factors that regulate skin pigmentation. Biofactors. 2009; 35:193-199.

7. Li X, Guo L, Sun Y, Zhou J, Gu Y, Li Y. Baicalein inhibits melanogenesis through activation of the ERK signaling pathway. Int J Mol Med. 2010; 25:923-927.

8. Kim DS, Kim SY, Chung JH, Kim KH, Eun HC, Park KC. Delayed ERK activation by ceramide reduces melanin synthesis in human melanocytes. Cell Signal. 2002; 14:779-785.

9. Schmuth M, Jiang YJ, Dubrac S, Elias PM, Feingold KR. Thematic review series: skin lipids. Peroxisome proliferator-activated receptors and liver $\mathrm{X}$ receptors in epidermal biology. J Lipid Res. 2008; 49:499-509.

10. Sertznig P, Reichrath J. Peroxisome proliferator-activated receptors (PPARs) in dermatology: challenge and promise. Dermatoendocrinology. 2011; 3:130-135.

11. Sertznig P, Seifert M, Tilgen W, Reichrath J. Peroxisome proliferator-activated receptors (PPARs) and the human skin: importance of PPARs in skin physiology and dermatologic diseases. Am J Clin Dermatol. 2008; 9:15-31. 
12. Rubins HB, Robins SJ, Collins D, Fye CL, Anderson JW, Elam MB, Faas FH, Linares E, Schaefer EJ, Schectman G, Wilt TJ, Wittes J. Gemfibrozil for the secondary prevention of coronary heart disease in men with low levels of highdensity lipoprotein cholesterol. Veterans Affairs HighDensity Lipoprotein Cholesterol Intervention Trial Study Group. N Engl J Med. 1999; 341:410-418.

13. Huang YC, Liu KC, Chiou YL, Yang CH, Chen TH, Li TT, Liu LL. Fenofibrate suppresses melanogenesis in B16F10 melanoma cells via activation of the p38 mitogenactivated protein kinase pathway. Chem Biol Interact. 2013; 205:157-164.

14. Fu J, Gaetani S, Oveisi F, Lo Verme J, Serrano A, Rodriguez De Fonseca F, Rosengarth A, Luecke H, Di Giacomo B, Tarzia G, Piomelli D. Oleylethanolamide regulates feeding and body weight through activation of the nuclear receptor PPAR-alpha. Nature. 2003; 425:90-93.

15. Guzman M, Lo Verme J, Fu J, Oveisi F, Blazquez C, Piomelli D. Oleoylethanolamide stimulates lipolysis by activating the nuclear receptor peroxisome proliferatoractivated receptor alpha (PPAR-alpha). J Biol Chem. 2004; 279:27849-27854.

16. Fu J, Oveisi F, Gaetani S, Lin E, Piomelli D. Oleoylethanolamide, an endogenous PPAR-alpha agonist, lowers body weight and hyperlipidemia in obese rats. Neuropharmacology. 2005; 48:1147-1153.

17. Yang LC, Guo H, Zhou H, Suo DQ, Li WJ, Zhou Y, Zhao Y, Yang WS, Jin X. Chronic oleoylethanolamide treatment improves spatial cognitive deficits through enhancing hippocampal neurogenesis after transient focal cerebral ischemia. Biochem Pharmacol. 2015; 94:270-281.

18. Zhou Y, Yang L, Ma A, Zhang X, Li W, Yang W, Chen C, Jin X. Orally administered oleoylethanolamide protects mice from focal cerebral ischemic injury by activating peroxisome proliferator-activated receptor alpha. Neuropharmacology. 2012; 63:242-249.

19. Yang L, Guo H, Li Y, Meng X, Yan L, Dan Z, Wu S, Zhou H, Peng L, Xie Q, Jin X. Oleoylethanolamide exerts anti-inflammatory effects on LPS-induced THP-1 cells by enhancing PPARalpha signaling and inhibiting the NF-kappaB and ERK1/2/AP-1/STAT3 pathways. Sci Rep. 2016; 6:34611.

20. Kono M, Dunn IS, Durda PJ, Butera D, Rose LB, Haggerty TJ, Benson EM, Kurnick JT. Role of the mitogen-activated protein kinase signaling pathway in the regulation of human melanocytic antigen expression. Mol Cancer Res. 2006; 4:779-792.

21. Elsalini OA, Rohr KB. Phenylthiourea disrupts thyroid function in developing zebrafish. Dev Genes Evol. 2003; 212:593-598.
22. Fu J, Kim J, Oveisi F, Astarita G, Piomelli D. Targeted enhancement of oleoylethanolamide production in proximal small intestine induces across-meal satiety in rats. Am J Physiol Regul Integr Comp Physiol. 2008; 295:R45-50.

23. Shibahara S, Yasumoto K, Amae S, Udono T, Watanabe K, Saito H, Takeda K. Regulation of pigment cell-specific gene expression by MITF. Pigment Cell Res. 2000; 13:98-102.

24. Sehgal VN, Verma P, Srivastava G, Aggarwal AK, Verma S. Melasma: treatment strategy. J Cosmet Laser Ther. 2011; $13: 265-279$.

25. Gaggioli C, Busca R, Abbe P, Ortonne JP, Ballotti R. Microphthalmia-associated transcription factor (MITF) is required but is not sufficient to induce the expression of melanogenic genes. Pigment Cell Res. 2003; 16:374-382.

26. Willson TM, Brown PJ, Sternbach DD, Henke BR. The PPARs: from orphan receptors to drug discovery. J Med Chem. 2000; 43:527-550.

27. Jang JY, Lee JH, Kang BW, Chung KT, Choi YH, Choi BT. Dichloromethane fraction of Cimicifuga heracleifolia decreases the level of melanin synthesis by activating the ERK or AKT signaling pathway in B16F10 cells. Exp Dermatol. 2009; 18:232-237.

28. Khaled M, Larribere L, Bille K, Ortonne JP, Ballotti R, Bertolotto C. Microphthalmia associated transcription factor is a target of the phosphatidylinositol-3-kinase pathway. $\mathrm{J}$ Invest Dermatol. 2003; 121:831-836.

29. Bellei B, Maresca V, Flori E, Pitisci A, Larue L, Picardo M. p38 regulates pigmentation via proteasomal degradation of tyrosinase. J Biol Chem. 2010; 285:7288-7299.

30. Saha B, Singh SK, Sarkar C, Bera R, Ratha J, Tobin DJ, Bhadra R. Activation of the Mitf promoter by lipidstimulated activation of p38-stress signalling to CREB. Pigment Cell Res. 2006; 19:595-605.

31. Park SY, Jin ML, Kim YH, Kim Y, Lee SJ. Aromaticturmerone inhibits alpha-MSH and IBMX-induced melanogenesis by inactivating CREB and MITF signaling pathways. Arch Dermatol Res. 2011; 303:737-744.

32. Chou ST, Chang WL, Chang CT, Hsu SL, Lin YC, Shih Y. Cinnamomum cassia essential oil inhibits $\alpha$-MSH-induced melanin production and oxidative stress in murine B16 melanoma cells. Int J Mol Sci. 2013; 14:19186-19201.

33. Choi TY, Kim JH, Ko DH, Kim CH, Hwang JS, Ahn S, Kim SY, Kim CD, Lee JH, Yoon TJ. Zebrafish as a new model for phenotype-based screening of melanogenic regulatory compounds. Pigment Cell Res. 2007; 20:120-127. 